Binghamton University

The Open Repository @ Binghamton (The ORB)

2016

\title{
Magnetoelastic Beam with Extended Polymer For Low Frequency Vibration Energy Harvesting
}

\author{
Alwathiqbellah Ibrahim \\ Binghamton University--SUNY \\ Shahrzad Towfighian \\ Binghamton University--SUNY, stowfigh@binghamton.edu \\ Mohammad I. Younis \\ Binghamton University--SUNY \\ Quang Su \\ Binghamton University--SUNY
}

Follow this and additional works at: https://orb.binghamton.edu/mechanical_fac

Part of the Mechanical Engineering Commons

\section{Recommended Citation \\ Ibrahim, Alwathiqbellah; Towfighian, Shahrzad; Younis, Mohammad I.; and Su, Quang, "Magnetoelastic Beam with Extended Polymer For Low Frequency Vibration Energy Harvesting" (2016). Mechanical Engineering Faculty Scholarship. 15. \\ https://orb.binghamton.edu/mechanical_fac/15}

This Conference Proceeding is brought to you for free and open access by the Mechanical Engineering at The Open Repository @ Binghamton (The ORB). It has been accepted for inclusion in Mechanical Engineering Faculty Scholarship by an authorized administrator of The Open Repository @ Binghamton (The ORB). For more information, please contact ORB@binghamton.edu. 


\title{
Magnetoelastic beam with extended polymer for low frequency vibration energy harvesting
}

\author{
Alwathiqbellah Ibrahim $^{\mathrm{a}}$, Shahrzad Towfighian ${ }^{\mathrm{b}}$, Mohammad Younis ${ }^{\mathrm{c}}$, and Quang $\mathrm{Su}^{\mathrm{d}}$ \\ Binghamton University, 4400 Vestal Parkway E., Binghamton, NY 13902
}

\begin{abstract}
Ambient energy in the form of mechanical kinetic energy is mostly considered waste energy. The process of scavenging and storing such energy is known as energy harvesting. Energy harvesting from mechanical vibration is performed using resonant energy harvesters $(\mathrm{EH})$ with two major goals: enhancing the power scavenged at low frequency sources of vibrations, and increasing the efficiency of scavenging energy by increasing the bandwidth near the resonant frequency. Toward such goals, we propose a piezoelectric EH of a composite cantilever beam with a tip magnet facing another magnet at a distance. The composite cantilever consists of a piezoelectric bimorph with an extended polymer material. With the effect of the nonlinearity of the magnetic force, higher amplitude can be achieved because of the generated bi-stability oscillations of the cantilever beam under harmonic excitation. The contribution of the this paper is to demonstrate lowering the achieved resonant frequency down to $17 \mathrm{~Hz}$ compared to $100 \mathrm{~Hz}$ for the piezoelectric bimorph beam without the extended polymer. Depending on the magnetic distance, the beam responses are divided to mono and bi-stable regions, for which we investigate static and dynamic behaviors. The dynamics of the system and the frequency and voltage responses of the beam are obtained using the shooting method.
\end{abstract}

Keywords: piezoelectric energy harvesting devices, magnetic force, bi-stability

\section{INTRODUCTION}

Ambient energy surrounds us in different forms, such as wind, solar, thermal and mechanical energy. Many of these are considered waste energy from the naturally generated sources. The process of scavenging and storing such energy is known as energy harvesting.

One of the major sources of energy for harvesting purposes is the prevalent mechanical vibrations present in the environment and transportation vehicles. Vibration energy harvested from these sources can be useful for autonomous powering of wireless sensors. ${ }^{1-6}$ Vibration energy harvesters are mechanical resonators that oscillate when attached to a vibration source. This oscillation is then converted to electric energy using electromagnetics, electrostatic or piezoelectric ${ }^{7}$ transducers. The most common transducer is the piezoelectric mechanism ${ }^{8-15}$ because of its energy density.

Linear resonators are inefficent because of their narrow freqeuncy bandwidth compared to the broadband ambient vibrations. Several methods for tuning of the resonator frequency with the dominant ambient frequency were explored including the use of magnets, ${ }^{16}$ but did not change the nature of narrow bandwidth. To solve the bandwidth problem, several nonlinear resonators were investigated during the past few years. Nonlinear systems have higher bandwidth oscillations which in turn increase the efficincy of the harvesting energy from broadband vibrations. Using magnetic nonlinearity, Mann et al. ${ }^{17}$ investigated the widening of frequency bandwidth by hardening effect and the increasing of the output power. Researchers studied the repulsive magnetic force effect on making a bi-stable resonator that showed a wide frequency response. ${ }^{18-22}$ The variation of the natural frequency and voltage output with the distance between the permanent magnets were explored experimentally by Ferrari et al. ${ }^{20-22}$ Barton et al. ${ }^{23}$ used electromagnetic transducers with a bi-stable resonator and revealed the effect

a: E-mail: aibrahi4@binghamton.edu

b: Corresponding author: E-mail: stowfigh@binghamton.edu, Telephone: 16077775315

c: E-mail: myounis@binghamton.edu

d: E-mail: qsu@binghamton.edu 
of varying the load resistance on the frequency bandwidth. They introduced the idea of using superharmonic resonances as a harvesting method at low frequencies. Using three real white noise sources, Vocca et al. ${ }^{24}$ evaluated the performance of a nonlinear resonator. They reported the output voltage and power as the distance between the two magnets varies in the bi-stable resonator. Al-Ashtari et al. ${ }^{16}$ found that trivial distances have significant effect on the output voltage because of the influence on the mode shape of the beam. Tang et al. ${ }^{25,26}$ found the optimal distance to be at the transition from mono-stable to bi-stable regime. In a recent study, ${ }^{27}$ they used a magnetic oscillator instead of a fixed permanent magnet and showed that frequency bandwidth can double in that case. This paper presents a study of a magnetoelastic piezoelectric energy harvester, which employs magnetic force to control the stability and the frequency of the energy harvester. The magnetic force is expanded into Taylor series up to nine order of nonlinear terms. A Reduced Order Model with the Galerkin approach are used to develop a single degree of freedom system under the effect of the nonlinear terms. A Shooting Method (ShM)is used to extract the steady state response of the harvester. Softening and hardening nonlinear behaviors are investigated by controlling the distance between the two magnets. First we start by formulating the free vibration problem. Then we extract the static and eigenvalue problems. Next we formulate the dynamic problem through the reduced order model. After that the experimental setup are explained. Finally we discussed the results and conclusions.

\section{PROBLEM FORMULATION OF FREE VIBRATION}

The nonlinear resonator is composed of a piezoelectric cantilever beam with a tip magnet facing the same pole of another magnet (Figure 1). To increase the amplitude of the resonator and lower its natural frequency, we use a polymer material for the cantilever beam that is partially convered by piezoelectric laminates. The magnetic force acts as a nonlinear spring that makes the system bi-stable. There have been studies on modeling of this bi-stable resonator mostly focusing on lumped paramter modeling. ${ }^{20}$ However, lumped modeling is only an approximation for the system's response at frequencies close to its natural frequency. To accurately evaluate the response of the resonator for a wide range of frequency, we develop a continuous model of the system and determine the response to a harmonic base excitation of $\ddot{z}(t)=A \cos (\Omega t)$, where $\Omega$ is the frequency of excitation and $A$ is the amplitude. In Figure $1, d$ is the horizontal distance between two magnets, $L$ is the length of the beam, $W(x, t)$ is the transverse deflection of the beam, $R_{L}$ is the load resistanc, and $F_{\text {mag }}$ is the magnetic force. The tip magnet is modeled as a rigid body with mass $M$ and mass moment of inertia $J=\frac{1}{6} M L_{m}^{2}$ with a side length $L_{m}$.

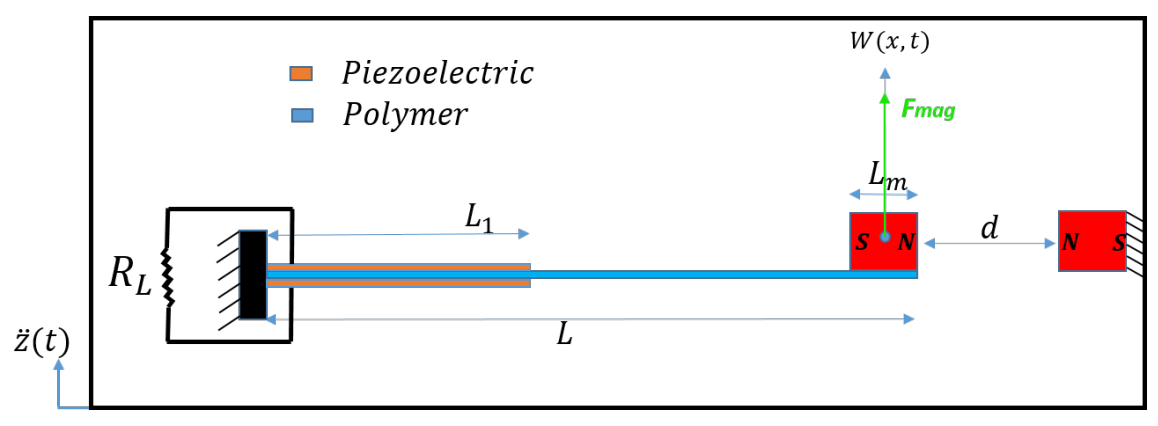

Figure 1: Schematic for the bi-stable resonator

The effect of the distance between magnets on the beam response and potential energy function is illustrated in Figure 2. Magnetic force is a function of separation distance and is responsible for creating one more equilibrium points to make a bi-stable system. The corresponding potential energy function is single well or double well depending on the distance between magnets. For large distances between magnets, the beam will oscillate around one stable point, point 1 (mono-stable oscillation). At small distances, the system will have two potential wells and the beam will oscillate between two stable equilibrium points, at points 2 and 3, (bi-stable oscillation). 


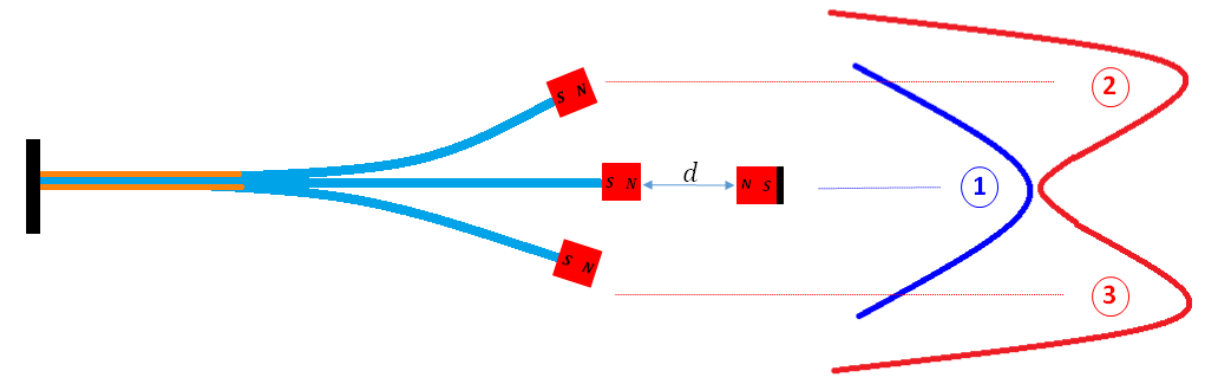

Figure 2: Mono-stable and bi-stable configurations of the resonator.

The energy harvester has two portions, the first part is the biomorph with the piezoelectric layer at the top and bottom $\left(0 \leq x \leq L_{1}\right)$, and the second part consists of a polymer layer $\left(L_{1} \leq x \leq L\right)$. By applying Newton's second law and considering the continuity conditions at $L_{1}$, the governing equations for free vibration (no base excitation) of the system and boundary conditions are obtained

$$
\begin{aligned}
& \rho A_{c} \ddot{W}_{1}(x, t)+E I_{c} W_{1 x x x x}(x, t)+c \dot{W}_{1}(x, t)+\vartheta V(t)\left[\frac{d \delta(x)}{d x}-\frac{d \delta(x-L)}{d x}\right]=0 \\
& \rho_{p} A_{p} \ddot{W}_{2}(x, t)+E_{p} I_{p} W_{2 x x x x}(x, t)+c \dot{W}_{2}(x, t)=0 \\
& C_{p} \dot{V}(t)+\left.\frac{\vartheta}{2} \frac{\partial \dot{W}(x, t)}{\partial x}\right|_{L_{1}}+\frac{V(t)}{R_{L}}=0
\end{aligned}
$$

where the third equation in Equation set (1) is for the voltage produced in the piezoelectric layer making a bi-stable resonator that showed a wide frequency response ${ }^{17-23}$

$$
\begin{aligned}
& W_{1}(0, t)=0, \quad W_{1 x}(0, t)=0, \quad W_{1}\left(L_{1}, t\right)-W_{2}\left(L_{1}, t\right)=0, \quad W_{1 x}\left(L_{1}, t\right)-W_{2 x}\left(L_{1}, t\right)=0 \\
& E I_{c} W_{1 x x}\left(L_{1}, t\right)-E_{p} I_{p} W_{2 x x}\left(L_{1}, t\right)=0, \quad E I_{c} W_{1 x x x}\left(L_{1}, t\right)-E_{p} I_{p} W_{2 x x x}\left(L_{1}, t\right)=0 \\
& E_{p} I_{p} W_{2 x x x}(L, t)-M \ddot{W}_{2}(L, t)-M L_{c} \ddot{W}_{2 x}(L, t)+F_{\text {magy }}-M g=0 \\
& E_{p} I_{p} W_{2 x x}(L, t)+M L_{c} \ddot{W}(L, t)+\left[M L_{c}^{2}+J\right] \ddot{W}_{2 x}(L, t)-L_{c} F_{\text {magy }}+L_{c} M g=0
\end{aligned}
$$

where $\rho A_{c}$ is the equivalent mass per unit length, for the biomorph part of the beam $\left(\rho_{1} A_{1}+\rho_{p} A_{p}+\rho_{2} A_{2}\right)$. The subscripts $(1,2)$ denote the biomorph and the polymer parts of the beam. $c$ is the damping, $\delta$ is the Dirac delta function, $E_{p}$ and $I_{p}$ are the polymer beam modulus of elasticity and second moment of inertia, respectively. $V$ and $R_{L}$ are the voltage and resistance load, respectively, while $C_{p}$ is the capacitance through piezoelectric layers. $M$ is the mass of the tip oscillating magnet, $E I_{c}$ and $\vartheta$ are the effective flexural rigidity of the biomorph part of the beam and the coupling coefficient term, respectively, and are given by

$$
\vartheta=e_{31} b\left(h_{p}+h_{1}\right), \quad E I_{c}=\frac{2 b}{3}\left\{E_{p} \frac{h_{p}^{3}}{8}+E_{1}\left[\left(h_{1}+\frac{h_{p}}{2}\right)^{3}-\frac{h_{p}^{3}}{8}\right]\right\}
$$

where $e_{31}$ is the piezoelectric constant, $b$ is the width of both the piezoelectric layer and the beam. $h_{1}$ and $h_{p}$ are the thickness of the piezoelectric layer and the polymer beam, respectively and $E_{1}$ is the Modulus of Elasticity of the piezoelectric layer. 
The total magnetic force between two dipoles is a function of spatial derivatives of their magnetic field, whose formula is given in reference. ${ }^{27}$ Total magnetic force can be written in terms of two components, One in the longitudinal direction which is assumed to balance the longitudinal stiffness of the beam, The other one in the transverse direction that is the component responsible for the beam transverse deflection and is given by:

$$
F_{\text {magy }}=\frac{F_{R} Y}{\left(Y^{2}+d^{2}\right)^{5 / 2}}
$$

where $F_{R}$ is the magnitude of the moments for magnetic dipoles and given by

$$
F_{R}=\frac{3 \varepsilon m_{1} m_{2}}{2 \pi}
$$

where $m_{1}$ and $m_{2}$ are the moments of magnetic dipoles for the fixed and tip magnets, respectively. $\varepsilon$ is the permeability of the free space and equal to $4 \pi \times 10^{-7} \frac{\mathrm{mkg}}{\mathrm{s}^{2} \mathrm{~A}^{2}}, d$ is the longitudinal separation distance between the centers of the two magnets, and $Y$ is the deflection of the center of the paddle in the transverse direction given by

$$
Y=W_{2}(L, t)+L_{c} W_{2 x}(L, t)
$$

As it can be seen from Equation (4), the magnetic force is a nonlinear function of beam tip displacement. This nonlinearity causes the system to have multiple equilibrium points.

\subsection{Static Problem}

The static problem can be formulated by putting all time derivatives to zero in Equations (1) and (2):

$$
\begin{gathered}
E I_{c} W_{s 1 x x x x}(x)=0, \quad E_{p} I_{p} W_{s 2 x x x x}(x)=0 \\
W_{s 1}(0)=0, \quad W_{s 1 x}(0)=0, \quad W_{s 1}\left(L_{1}\right)-W_{s 2}\left(L_{1}\right)=0, \quad W_{s 1 x}\left(L_{1}\right)-W_{s 2 x}\left(L_{1}\right)=0 \\
E I_{c} W_{s 1 x x}\left(L_{1}\right)-E_{p} I_{p} W_{s 2 x x}\left(L_{1}\right)=0, \quad E I_{c} W_{s 1 x x x}\left(L_{1}\right)-E_{p} I_{p} W_{s 2 x x x}\left(L_{1}\right)=0 \\
E_{p} I_{p} W_{s 2 x x x}(L)+F_{\text {magys }}-M g=0, \quad E_{p} I_{p} W_{s 2 x x}(L)-L_{c} F_{\text {magys }}+L_{c} M g=0
\end{gathered}
$$

where the subscript $s$ refer to static, and $F_{\text {magys }}$ is the static form of the magnetic force in the transverse direction and given by

$$
F_{\text {magys }}=\frac{F_{R} Y_{s}}{\left(Y_{s}^{2}+d^{2}\right)^{5 / 2}}
$$

where $Y_{s}$ is the static deflection for the magnetic tip center. Now the general solution for the static equilibrium equations (7) can be obtained by applying the static boundary conditions of Equations (8). 


\subsection{Eigenvalue Problem}

To determine the natural frequency and mode shapes of the system, we solve the eigenvalue problem. The total deflection of the beam can be linearized around its equilibrium points:

$$
W(x, t)=W_{s}(x)+u(x, t)
$$

where $W_{s}(x)$ and $u(x, t)$ are static equilibrium position and dynamic response, respectively. Substituting Equation (10) into Equation (6), we obtain the following form for the displacements for center of the magnet

$$
\begin{aligned}
Y & =W_{2 s}(L)+u_{2}(x, t)+L_{c} W_{2 s x}(L)+L_{c} u_{2 x}(x, t) \\
& =W_{2 s}(L)+L_{c} W_{2 s x}(L)+u_{2}(x, t)+L_{c} u_{2 x}(x, t) \\
& =Y_{s}+Y_{u}
\end{aligned}
$$

where $Y_{s}$ is the static displacement and $Y_{u}$ is the dynamic displacement of the magnet center. Substituting Equation (11) into the magnetic force Equation (4), and expanding with Taylor series around $Y_{u}=0$ and neglecting nonlinear terms yields

$$
\begin{aligned}
F_{\text {magy }} & =\frac{F_{R} Y s}{\left(d^{2}+Y_{s}^{2}\right)^{5 / 2}}+\frac{F_{R}\left(d^{2}-4 Y_{s}^{2}\right)}{\left(d^{2}+Y_{s}^{2}\right)^{7 / 2}} Y_{u} \\
& =F_{\text {magys }}+F_{\text {magyu }}
\end{aligned}
$$

where $F_{\text {magys }}$ and $F_{\text {magyu }}$ are the static and linearized dynamic magnetic forces, respectively. Now to find the mode shapes and natural frequencies at a given static equilibrium position, we assume a solution in the form of

$$
u_{j}(x, t)=\phi_{j}(x) e^{i \omega t}
$$

where $\phi_{j}(x)$ is the first mode shape, and the subscript $\mathrm{j}$ corresponds to the beam part. $\omega$ is the beam natural frequency. Neglecting all nonlinear, damping, and voltage terms and substituting Equations (13) and (11) into Equations (1) and canceling all the static terms from both sides of equation we can extract the eigenvalue problem as

$$
\phi_{1 x x x x}(x)-a_{1}^{4} \phi_{1}(x)=0, \quad \phi_{2 x x x x}(x)-a_{2}^{4} \phi_{2}(x)=0
$$

where

$$
a_{1}^{4}=\frac{\rho A_{c} \omega^{2}}{E I_{c}}, \quad a_{2}^{4}=\frac{\rho_{p} A_{p} \omega^{2}}{E_{p} I_{p}}
$$

and

$$
\begin{aligned}
& \phi_{1}(0)=0, \quad \phi_{1 x}(0)=0, \quad \phi_{1}\left(L_{1}\right)-\phi_{2}\left(L_{1}\right)=0, \quad \phi_{1 x}\left(L_{1}\right)-\phi_{2 x}\left(L_{1}\right)=0 \\
& E I_{c} \phi_{1 x x}\left(L_{1}\right)-E_{p} I_{p} \phi_{2 x x}\left(L_{1}\right)=0, \quad E I_{c} \phi_{1 x x x}\left(L_{1}\right)-E_{p} I_{p} \phi_{2 x x x}\left(L_{1}\right)=0 \\
& \phi_{2 x x}(L)-L_{c} \eta \phi_{2}(L)-\frac{5}{3} L_{c}^{2} \eta \phi_{2 x}(L)-\frac{L_{c}}{E_{p} I_{p}} F_{\text {magy } \phi}=0 \\
& \phi_{2 x x x}(L)+\eta \phi_{2}(L)+L_{c} \eta \phi_{2 x}(L)+\frac{1}{E_{p} I_{p}} F_{\text {magy }}=0
\end{aligned}
$$

where $J=\frac{2}{3} M L_{c}^{2}, \eta=\frac{M \omega^{2}}{E_{p} I_{p}}$, and $F_{m a g y \phi}$ is the magnetic force as a function of the mode shape and given by 


$$
F_{\text {magy } \phi}=\frac{F_{R}\left(d^{2}-4 Y_{s}^{2}\right)}{\left(d^{2}+Y_{s}^{2}\right)^{7 / 2}} Y_{\phi}
$$

where $Y_{\phi}$ is given by

$$
Y_{\phi}=\phi(L)+L_{c} \phi_{x}(L)
$$

\section{DYNAMIC PROBLEM AND REDUCED ORDER MODEL (ROM)}

To evaluate the dynamic response of the resonator and the produced voltage by the piezoelectric layer, the governing equations for the forced vibration are derived using Lagrange's equations. The total kinetic and potential energy for the energy harvester is divided into two parts, first part for the biomorph beam and the second part for the polymer beam. At the end, the two governing equation are merged into one governing equation in addition to the electrical equation of the piezoelectric layers.

For the first part of the beam (biomorph portion), the kinetic and potential energy are given by $T_{1}$ and $U_{1}$, respectively:

$$
\begin{gathered}
T_{1}=\int_{0}^{L_{1}} \frac{1}{2} \rho A_{c} \dot{W}_{1}^{2}(x, t) d x \\
U_{1}=\int_{0}^{L_{1}} \frac{1}{2} E I_{c} W_{1 x x}^{2}(x, t) d x-\frac{1}{2} \vartheta V(t) W_{1 x}\left(L_{1}, t\right)-\frac{1}{2} C_{p} V^{2}(t)
\end{gathered}
$$

where the last two terms in Equation (20) are the potential energy for the piezoelectric layers. For the second part of the beam (polymer layer), $T_{2}$ and $U_{2}$ are given by:

$$
\begin{gathered}
T_{2}=\int_{L_{1}}^{L} \frac{1}{2} \rho_{p} A_{P} \dot{W}_{2}^{2}(x, t) d x+\frac{1}{2} M\left[\dot{W}_{2}(L, t)+L_{c} \dot{W}_{2 x}(L, t)\right]^{2}+\frac{1}{2} J \dot{W}_{2 x}^{2}(L, t) \\
U_{2}=\int_{L_{1}}^{L} \frac{1}{2} E_{p} I_{p} W_{2 x x}^{2}(x, t) d x+U_{M a g}
\end{gathered}
$$

where, $U_{M a g}$ is the magnetic potential energy. The total kinetic energy is given by $T=T_{1}+T_{2}$, while the total potential energy is given by $U=U_{1}+U_{2}$. Lagrange's Equation is written as $\ell=T-U$. To develop a ROM for the energy harvester, we need to express the transverse deflection using Galerkin approach as:

$$
\begin{array}{ll}
W_{1}(x, t)=W_{1 s}(x)+\sum_{i=1}^{n} \phi_{1 i}(x) q_{i}(t)+Z(t), & 0 \leq x \leq L_{1} \\
W_{2}(x, t)=W_{2 s}(x)+\sum_{i=1}^{n} \phi_{2 i}(x) q_{i}(t)+Z(t), & L_{1} \leq x \leq L
\end{array}
$$


where $q_{i}(t)$ is the modal coordinate, $Z(t)$ is the base motion, and $n$ is the number of mode shapes. Using Lagrange's Equation with one mode approximation, one can obtain

$$
\begin{gathered}
\ddot{q}_{1}(t)+2 \mu \omega_{n} \dot{q}_{1}(t)+\omega_{n}^{2} q_{1}(t)-\chi V(t)-F_{0} \cos (\Omega t)+\frac{1}{M_{33}} F_{\text {magyu }}=0 \\
\dot{V}(t)+\kappa \dot{q}_{1}(t)+\lambda V(t)=0
\end{gathered}
$$

where

$$
\begin{gathered}
\mu=\frac{D_{33}}{2 M_{33} \omega_{n}}, \quad \omega_{n}=\sqrt{\frac{K_{33}+\alpha_{1}}{M_{33}}, \quad \chi=\frac{\theta_{33}}{M_{33}}} \\
F_{0}=\frac{A \Omega^{2} H_{33}}{M_{33}}, \quad \lambda=\frac{1}{C_{p} R_{L}}, \quad \kappa=\frac{\theta_{33}}{C_{p}} \\
M_{33}=\int_{0}^{L_{1}} \rho A_{c} \phi_{1}^{2}(x) d x+\int_{L_{1}}^{L} \rho_{p} A_{p} \phi_{2}^{2}(x) d x+M\left(\phi_{2}(L)+L_{c} \phi_{2 x}(L)\right)^{2}+J \phi_{2 x}^{2}(L) \\
D_{33}=c \int_{0}^{L_{1}} \phi_{1}^{2}(x) d x+c \int_{L_{1}}^{L} \phi_{2}^{2}(x) d x \\
K_{33}=\int_{0}^{L_{1}} E I_{c} \phi_{1 x x}^{2}(x) d x+\int_{L_{1}}^{L} E_{p} I_{p} \phi_{2 x x}^{2}(x) d x \\
H_{33}=\int_{0}^{L_{1}} \rho A_{c} \phi_{1}(x) d x+M\left(\phi_{2}(L)+L_{c} \phi_{2 x}(L)\right)+\int_{L_{1}}^{L} \rho_{p} A_{p} \phi_{2}(x) d x \\
F_{\text {magyu }}=\alpha_{2} q_{1}^{2}(t)+\alpha_{3} q_{1}^{3}(t)+\alpha_{4} q_{1}^{4}(t)+\alpha_{5} q_{1}^{5}(t)+\alpha_{6} q_{1}^{6}(t)+\alpha_{7} q_{1}^{7}(t)+\alpha_{8} q_{1}^{8}(t)+\alpha_{9} q_{1}^{9}(t)+O\left(q_{1}^{10}\right)
\end{gathered}
$$

where $\alpha_{1}, \ldots, \alpha_{9}$ are the coefficients due to the expansion with Taylor series. To solve the above dynamic equations, we use the shooting method as explained in reference. ${ }^{28}$ Toward this we let

$$
\begin{aligned}
X_{1} & =q_{1}(t) \\
X_{2} & =\dot{q}_{1}(t) \\
X_{3} & =V(t)
\end{aligned}
$$

This will introduce the following three first order ode: 


$$
\begin{aligned}
& \dot{X}_{1}=X_{2} \\
& \dot{X}_{2}=-\left(2 \mu \omega_{n} X_{2}+\omega_{n}^{2} X_{1}-\chi X_{3}+F_{0} \cos (\Omega t)+\frac{1}{M_{33}} F_{\text {maguX }}\right) \\
& \dot{X}_{3}=-\left(\kappa X_{2}+\lambda X_{3}\right)
\end{aligned}
$$

Where $F_{\text {maguX }}$ is the magnetic force but written in state space form up to the ninth order term and given by

$$
F_{\text {magy } X}=\alpha_{2} X_{1}^{2}(t)+\alpha_{3} X_{1}^{3}(t)+\alpha_{4} X_{1}^{4}(t)+\alpha_{5} X_{1}^{5}(t)+\alpha_{6} X_{1}^{6}(t)+\alpha_{7} X_{1}^{7}(t)+\alpha_{8} X_{1}^{8}(t)+\alpha_{9} X_{1}^{9}(t)
$$
by

The appropriate initial conditions that will give a periodic solution for the first order ode system are given

$$
\begin{aligned}
& q_{1}(0)=X_{1}(0)=\eta_{1} \\
& \dot{q}_{1}(0)=X_{2}(0)=\eta_{2} \\
& V(0)=X_{3}(0)=\eta_{3}
\end{aligned}
$$

Introduce a new variables by taking the derivative of each variable, $\left(X_{1}, X_{2}, X_{3}\right)$, with respect to the three initial conditions, $\left(\eta_{1}, \eta_{2}, \eta_{3}\right)$, and following procedure in the reference ${ }^{28}$ we can construct the following first order ode system:

$$
\begin{aligned}
& \dot{X}_{4}=X_{7} \\
& \dot{X}_{5}=X_{8} \\
& \dot{X}_{6}=X_{9} \\
& \dot{X}_{7}=-\left(2 \mu \omega_{n} X_{7}+\omega_{n}^{2} X_{4}-\chi X_{10}+\frac{X_{4}}{M_{33}} \frac{\partial F_{\text {magy } X}}{\partial X_{1}}\right) \\
& \dot{X}_{8}=-\left(2 \mu \omega_{n} X_{8}+\omega_{n}^{2} X_{5}-\chi X_{11}+\frac{X_{5}}{M_{33}} \frac{\partial F_{\text {magy }}}{\partial X_{1}}\right) \\
& \dot{X}_{9}=-\left(2 \mu \omega_{n} X_{9}+\omega_{n}^{2} X_{6}-\chi X_{12}+\frac{X_{6}}{M_{33}} \frac{\partial F_{\text {magy }}}{\partial X_{1}}\right) \\
& \dot{X}_{10}=-\left(\kappa X_{7}+\lambda X_{10}\right) \\
& \dot{X}_{11}=-\left(\kappa X_{8}+\lambda X_{11}\right) \\
& \dot{X}_{12}=-\left(\kappa X_{9}+\lambda X_{12}\right)
\end{aligned}
$$

where

$$
\frac{\partial F_{\text {magy } X}}{\partial X_{1}}=2 \alpha_{2} X_{1}(t)+3 \alpha_{3} X_{1}^{2}(t)+4 \alpha_{4} X_{1}^{3}(t)+5 \alpha_{5} X_{1}^{4}(t)+6 \alpha_{6} X_{1}^{5}(t)+7 \alpha_{7} X_{1}^{6}(t)+8 \alpha_{8} X_{1}^{7}(t)+9 \alpha_{9} X_{1}^{8}(t)
$$

The initial conditions for the first order ode system in Equations (38) are given by

$$
\begin{aligned}
& X_{1}(0)=\eta_{10}, \quad X_{2}(0)=\eta_{20}, \quad X_{3}(0)=\eta_{30} \\
& X_{4}(0)=1, \quad X_{5}(0)=0, \quad X_{6}(0)=0 \\
& X_{7}(0)=0, \quad X_{8}(0)=1, \quad X_{9}(0)=0 \\
& X_{10}(0)=0, \quad X_{11}(0)=0, \quad X_{12}(0)=1
\end{aligned}
$$


where $\left(\eta_{10}, \eta_{20}, \eta_{30}\right)$ are the initial guesses for the initial conditions that will lead to periodic solution. Integrate numerically Equations (35), (38) and Equations (40) over one period $T, T=\frac{2 \pi}{\Omega}$, where $\Omega$ is the excitation frequency. After that the values $X_{4}-X_{12}$ are calculated at time T, and the error in the initial conditions are calculated and updated by the following algebraic equation:

$$
\left\{\left(\begin{array}{ccc}
X_{4} & X_{5} & X_{6} \\
X_{7} & X_{8} & X_{9} \\
X_{10} & X_{11} & X_{12}
\end{array}\right)-[I]\right\}\left\{\begin{array}{l}
\partial \eta_{1} \\
\partial \eta_{2} \\
\partial \eta_{3}
\end{array}\right\}=\left\{\begin{array}{l}
\eta_{10}-X_{1}\left(T, \eta_{10}, \eta_{20}, \eta_{30}\right) \\
\eta_{20}-X_{2}\left(T, \eta_{10}, \eta_{20}, \eta_{30}\right) \\
\eta_{30}-X_{3}\left(T, \eta_{10}, \eta_{20}, \eta_{30}\right)
\end{array}\right\}
$$

where, $I$ is the Identity matrix, $\left(\partial \eta_{1}, \partial \eta_{2}, \partial \eta_{3}\right)$ are the errors in the initial conditions guesses. The process is repeated until convergence achieved and the errors are minimized. Then the stability analyzed for the periodic solution obtained from the previous step. Now we solve for the eigenvalues, called Floquet multipliers, of the Manodromy matrix shown below:

$$
\left(\begin{array}{ccc}
X_{4}(T) & X_{5}(T) & X_{6}(T) \\
X_{7}(T) & X_{8}(T) & X_{9}(T) \\
X_{10}(T) & X_{11}(T) & X_{12}(T)
\end{array}\right)
$$

Three Floquet multipliers will be the output of the eigenvalues for the Manodromy matrix, and the stability is analyzed according to the next equation:

$$
\text { Stability }= \begin{cases}\text { Abs }(\text { Floquet multipliers })<1 & \text { Stable } \\ \text { Otherwise } & \text { Unstable }\end{cases}
$$

\section{EXPERIMENTAL SETUP}

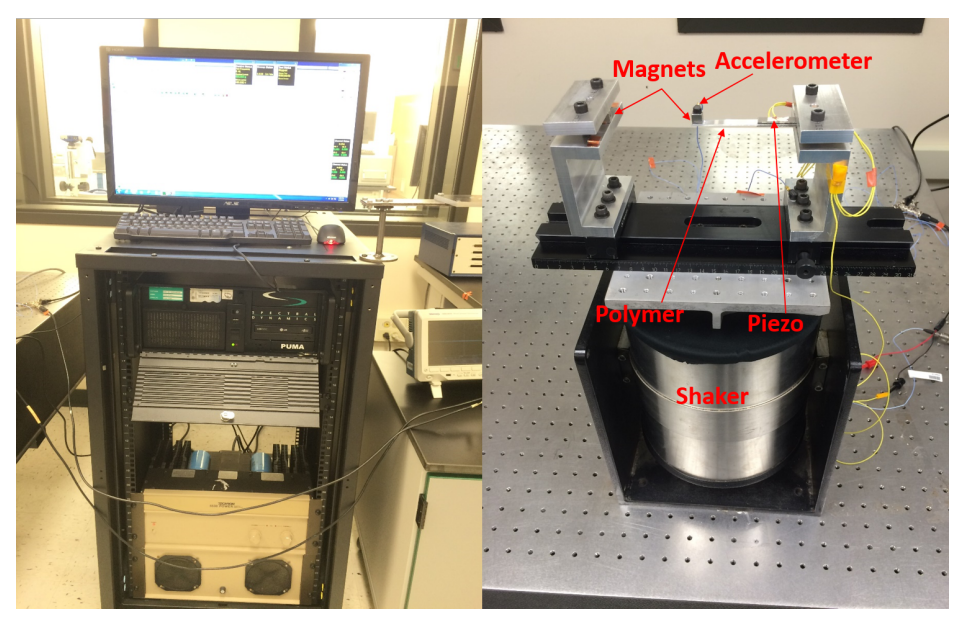

Figure 3: The experimental setup of the piezoelectric EH, cantilever harvester to the right and PUMA Spectral Dynamics Analyzer to the left.

Figure 3 shows the experimental setup used to test the magnetoelastic energy harvester. The setup consist of the PUMA Spectral Dynamics Analyzer(PSDA) and and the resonator attached to a rigid base with a rail to adjust the initial magnet distance. The resonator and its base were placed on a shaker to apply the base vibration. Materials used included a polymeric beam mad of Makrolon polycarbonate with modulus of elasticity of $2.34 \mathrm{GPa}$, partially covered by the piezoelectric material, Lead zirconate titanate (PZT)-5A Navy Type II with modules of elasticity of $66 \mathrm{GPa}$ and piezoelectric voltage constant of $\epsilon_{31}=-11 \times 10^{-3} \mathrm{Vm} / \mathrm{N}$, at the 
top and bottom. Cubic magnets with side length of $8 \mathrm{~mm}$ and magnetic moment of $0.5 \mathrm{~A}^{2} / \mathrm{m}$. The rest of the parameters are listed in Table 1. Two accelerometers; PCB Piezotronics LW149104 and LW196600; with sensitivities of $10.28 \mathrm{mV} / \mathrm{g}$ and $98 \mathrm{mV} / \mathrm{g}$, will be used to measure the output and input acceleration signals, receptively. The accelerometer with higher sensitivity will be placed on the rigid holder while the accelerometer with lower sensitivity will be placed on the beam tip magnet. Both accelerometers were connected to acceleration data logger, which in turn was connected to the PSDA. The data logger had many channels to receive the data from the accelerometers and the piezoelectric circuit. The PSDA sent the signal to the amplifier, which amplified the signal to vibrate the shaker at the specified excitation amplitude for a required range of excitation frequencies.

Table 1: Geometrical and material properties of the harvester.

\begin{tabular}{|c|c|c|c|}
\hline Parameters & Polymer & PZR 5A & Magnet \\
\hline Length & $7.2 \mathrm{~cm}$ & $3 \mathrm{~cm}$ & $8.0 \mathrm{~mm}$ \\
\hline Width & $1.0 \mathrm{~cm}$ & $1.0 \mathrm{~cm}$ & $8.0 \mathrm{~mm}$ \\
\hline Thickness & $1.0 \mathrm{~mm}$ & $1.0 \mathrm{~mm}$ & $8.0 \mathrm{~mm}$ \\
\hline Density & $1220 \mathrm{~kg} / \mathrm{m}^{3}$ & $7800 \mathrm{~kg} / \mathrm{m}^{3}$ & $7500 \mathrm{~kg} / \mathrm{m}^{3}$ \\
\hline Modulus & $2.344 \mathrm{GPa}$ & $66 \mathrm{GPa}$ & - \\
\hline Magnetic moment & - & - & $0.5 A^{2} / \mathrm{m}$ \\
\hline Piezoelectric constant & - & -3 & - \\
\hline
\end{tabular}

\section{RESULTS AND DISCUSSIONS}

Using the experimental setup in Figure 3, the frequency response curve and the frequency voltage curve were first measured for the linear resonator (for large distance between magnets) under $0.5 \mathrm{~g}$ base excitation amplitude (Figures 4a and 4b). Figure 4a shows the experimental and simulated results for the deflection of the tip magnet center. The resonance frequency was found to be $17.45 \mathrm{~Hz}$ at maximum peak-to-peak deflection of $9.5 \mathrm{~mm}$. Figure $4 \mathrm{~b}$ shows the corresponding output voltage, with a maximum peak-to-peak of $2.4 \mathrm{~V}$. Simulations using shooting method and measured results are in good agreements. It should be noted that the beam exclusively made of piezoelectric laminate with the same length has the the natural frequency of around $100 \mathrm{~Hz}$ compared to $17.45 \mathrm{~Hz}$ for the proposed beam with extended polymer. That means that our harvester lowered the natural frequency by $87 \%$. Operating the resonator at smaller distances between magnets, frequency broadening is achieved as explained next.

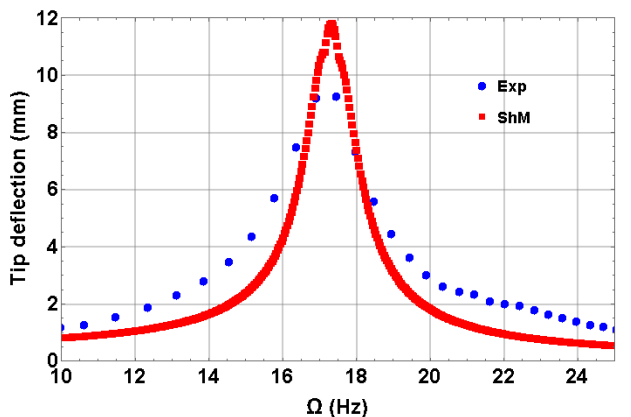

(a)

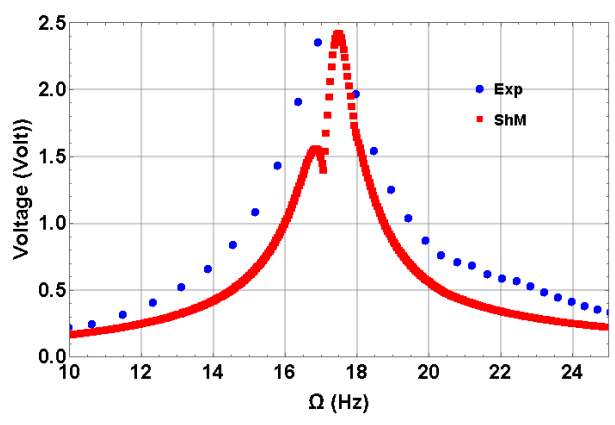

(b)

Figure 4: (a) Frequency response curve for the linear resonator. (b) Frequency voltage curve for the linear resonator. Excitation Amplitude $A=0.5 g$, Damping $\mu=0.0364$.

Figure 5 shows the variation of the static deflection for the center of the tip magnet with the distance between two magnets. Maximum deflections were obtained was $20 \mathrm{~mm}$. From the static response it's clearly shown that there is a critical separation distance between the two magnets, called threshold separation distance $d_{t h}$ which has a value of $20 \mathrm{~mm}$. This threshold value divided the static response into two major regions. The first region $\left(d>d_{t h}\right)$ is called mono-stable region, where the system oscillates around one stable equilibrium 
point in the middle. The second region $\left(d<d_{t h}\right)$ is called bi-stable region, where the system oscillates between two equilibrium stable points. The static profile contains one stable branch for the mono-stable regime and three branches for the bi-stable regime; upper and lower branches are stable, while the middle one is unstable. The phenomena of symmetry breaking bifurcation is shown clearly at the threshold value. The reason for this breaking is the weight of system that acts as a constant force in the static equilibrium equation in addition to the nonlinearity caused by the magnetic force.

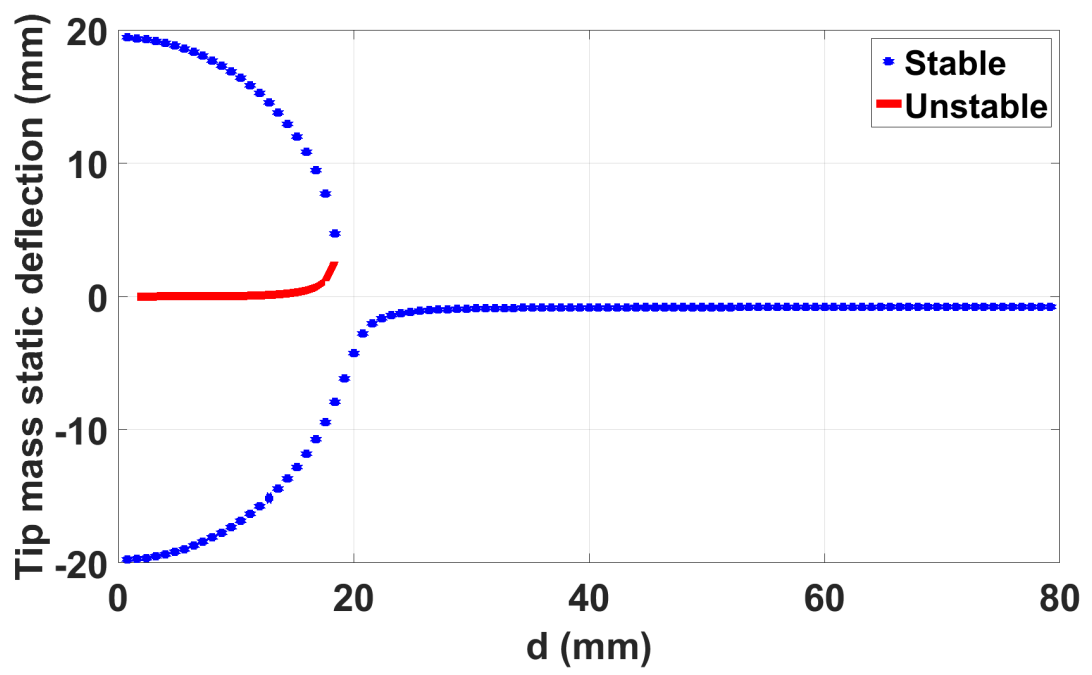

Figure 5: Simulated static response of the center of the tip magnet as a function of the distance between two magnets $(d)$. Threshold distance, $d_{t h}$ is found to be $20 \mathrm{~mm}$.

The dynamics of the energy harvester in the Mono-Stable region is explored for a separation distance of $40 \mathrm{~mm}$ between the two magnets. Figure 6 shows the experimental and simulated displacement and the voltage responses under $0.5 \mathrm{~g}$ excitation level. Simulations using shooting method shows a good agreement with the experimental results.

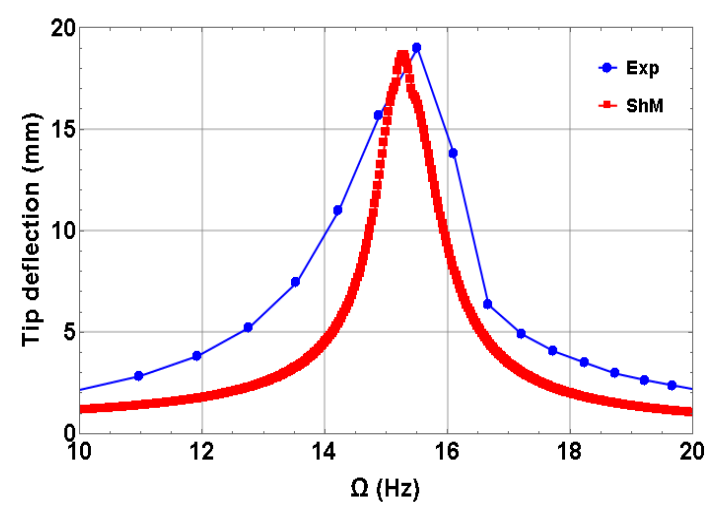

(a)

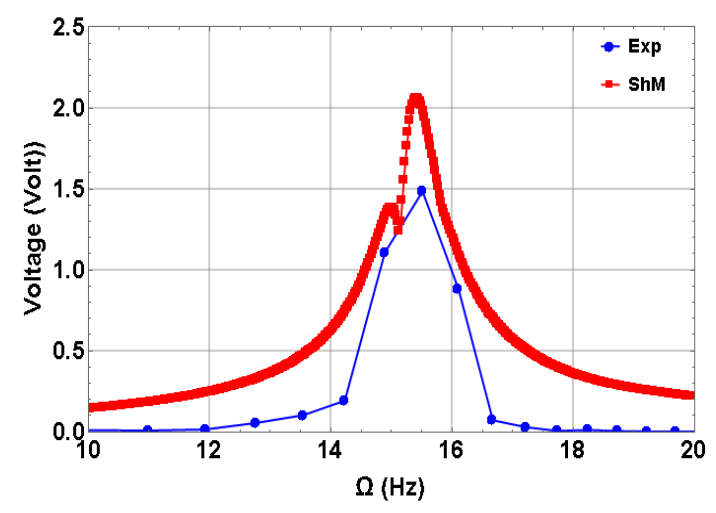

(b)

Figure 6: Mono-Stable results for the nonlinear energy harvester at $d=40 \mathrm{~mm}, A=0.5 \mathrm{~g}$ and damping $\mu=0.0364$ (a) Frequency response curves . (b) Frequency voltage curves.

Figure 7 shows the experimental and simulated displacement and the voltage responses under $0.5 \mathrm{~g}$ excitation level in the bi-stable region at separation distance of $18 \mathrm{~mm}$ between the two magnets. It is noted that the frequency softening that occurs in the bi-stable region is closely simulated by the shooting method. 


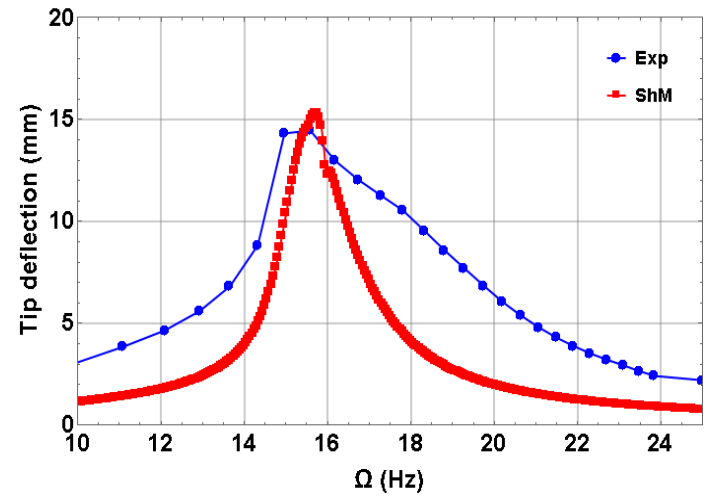

(a)

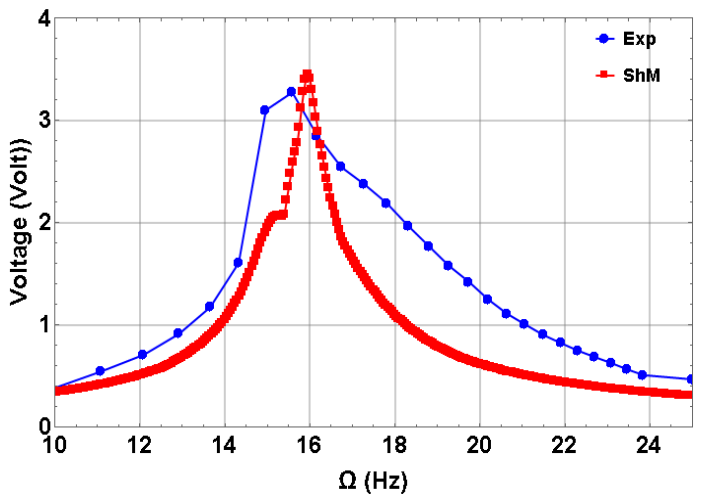

(b)

Figure 7: Bi-stable results for the nonlinear energy harvester at $d=18 \mathrm{~mm}, A=0.5 \mathrm{~g}$ and damping $\mu=0.0364$ (a) Frequency response curves . (b) Frequency voltage curves.

The resonator shows interesting behavior at distances close to the threshold distance. Figure 8 shows the experimental results at the mono-stable region for $d=22 \mathrm{~mm}$, close to the threshold. Higher bandwidth is achieved due to the hardening phenomenon. Figure $8 \mathrm{~b}$ is the corresponding voltage response curve. The highest voltage at excitation level of $0.5 \mathrm{~g}$ was measured peak to peak as $3.3 \mathrm{~V}$.

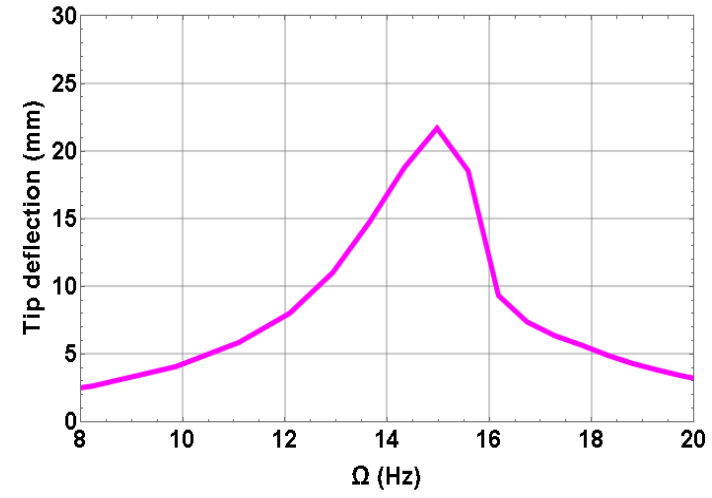

(a)

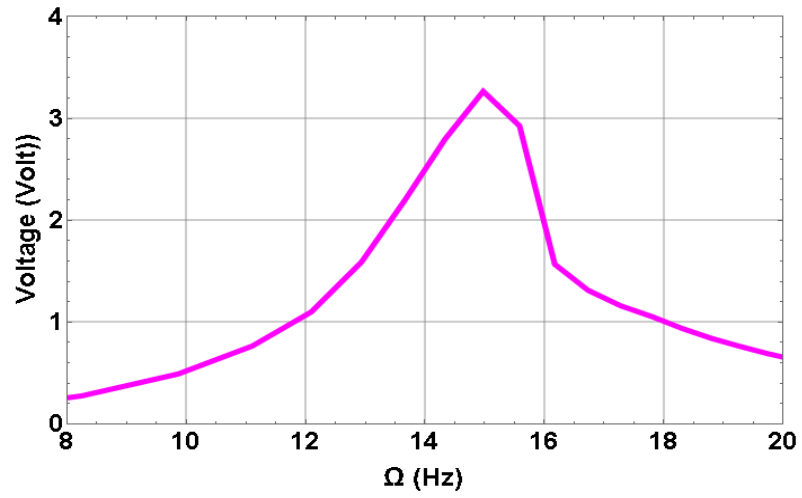

(b)

Figure 8: Mono-stable results for the nonlinear energy harvester at $d=22 \mathrm{~mm}, A=0.5 \mathrm{~g}$ (a) Frequency response curves . (b) Frequency voltage curves.

The dynamics of the energy harvester in the bi-stable region was also explored at $d=18 \mathrm{~mm}$, close to the threshold distance between two magnets. Figure 9 shows the experimental results at the excitation level of $0.5 \mathrm{~g}$. In contrast to the mono-stable region, the increase in the frequency bandwidth is resulted from frequency softening. 


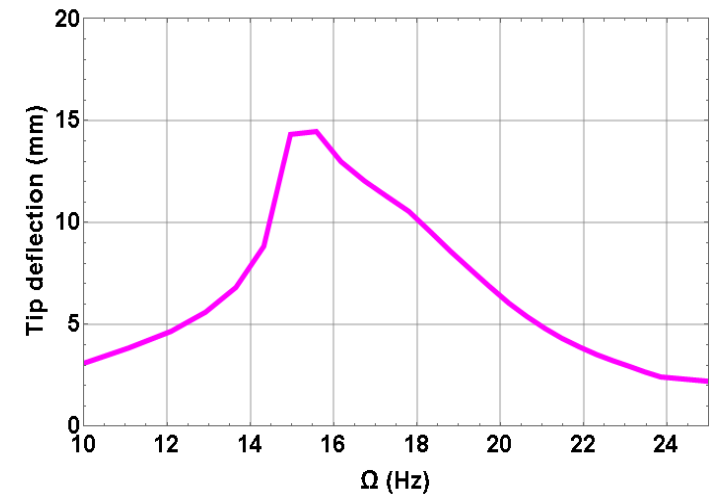

(a)

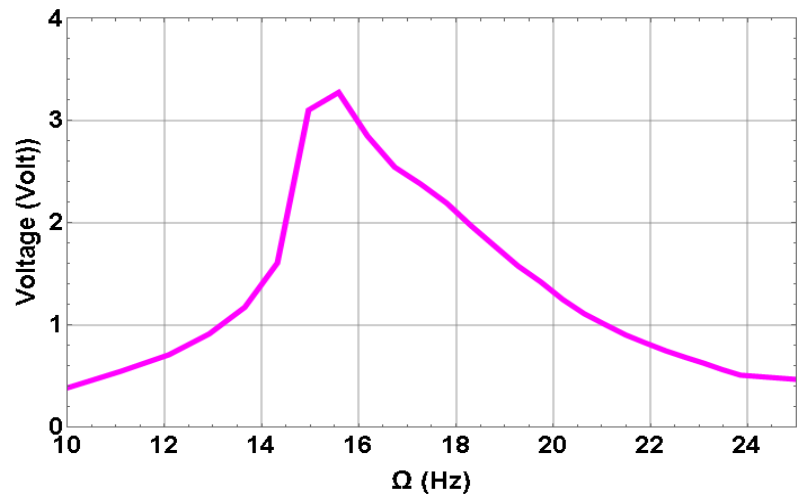

(b)

Figure 9: Bi-stable results for the nonlinear energy harvester at $d=18 \mathrm{~mm}, A=0.5 \mathrm{~g}$ (a) Frequency response curves . (b) Frequency voltage curves.

Comparing the output voltage for the nonlinear resonator close to the threshold distance (Figures $6 \mathrm{~b}$ and $9 \mathrm{~b}$ ) with the linear resonator at a large distance (Figure 4a), one can conclude that the output voltage has increased by $37 \%$.

\section{CONCLUSIONS}

A magnetoelastic piezoelectric energy harvester was investigated in this paper to increase the frequency bandwidth and amplitude of the response. The natural frequency of the resonator energy harvester was decreased from $100 \mathrm{~Hz}$ to $17 \mathrm{~Hz}$ using an extended polymer layer, which makes it more suitable for energy harvesting from ambient vibrations. A mathematical model for the continuous system of the energy harvester was developed considering the first mode shape of the resonator. Static analysis of the harvester revealed a threshold value that divided the nature of the response to mono- and bi-stable regimes based on the distance between two magnets. Dynamic responses of the system were measured in both mono-stable and bi-stable regions. Using the mathematical model, numerical method of shooting was used to solve the governing equations. Widening of frequency bandwidth was achieved by frequency hardening and softening at mono-stable and bi-stable regions, respectively. Compared to a linear resonator, an increment of $37 \%$ in the output voltage was obtained at distances close to the threshold distance for the nonlinear resonator.

\section{REFERENCES}

[1] Roundy, S., Wright, P. K., and Rabaey, J., "A study of low level vibrations as a power source for wireless sensor nodes," Computer communications 26(11), 1131-1144 (2003).

[2] Mitcheson, P. D., Green, T. C., Yeatman, E. M., and Holmes, A. S., "Architectures for vibration-driven micropower generators," Microelectromechanical Systems, Journal of 13(3), 429-440 (2004).

[3] Cook-Chennault, K., Thambi, N., and Sastry, A., "Powering mems portable devicesa review of nonregenerative and regenerative power supply systems with special emphasis on piezoelectric energy harvesting systems," Smart Materials and Structures 17(4), 043001 (2008).

[4] González, J. L., Rubio, A., and Moll, F., "Human powered piezoelectric batteries to supply power to wearable electronic devices," International journal of the Society of Materials Engineering for Resources 10(1), 34-40 (2002).

[5] Mathúna, C. Ó., ODonnell, T., Martinez-Catala, R. V., Rohan, J., and OFlynn, B., "Energy scavenging for long-term deployable wireless sensor networks," Talanta 75(3), 613-623 (2008).

[6] Torah, R., Glynne-Jones, P., Tudor, M., O’Donnell, T., Roy, S., and Beeby, S., "Self-powered autonomous wireless sensor node using vibration energy harvesting," Measurement Science and Technology 19(12), $125202(2008)$. 
[7] Beeby, S. P., Tudor, M. J., and White, N., "Energy harvesting vibration sources for microsystems applications," Measurement science and technology 17(12), R175 (2006).

[8] Mitcheson, P. D., Yeatman, E. M., Rao, G. K., Holmes, A. S., and Green, T. C., "Energy harvesting from human and machine motion for wireless electronic devices," Proceedings of the IEEE 96(9), 1457-1486 (2008).

[9] Sterken, T., Baert, K., Van Hoof, C., Puers, R., Borghs, G., and Fiorini, P., "Comparative modelling for vibration scavengers [mems energy scavengers]," in [Sensors, 2004. Proceedings of IEEE], 1249-1252, IEEE (2004).

[10] James, E., Tudor, M., Beeby, S., Harris, N., Glynne-Jones, P., Ross, J., and White, N., "An investigation of self-powered systems for condition monitoring applications," Sensors and Actuators A: Physical 110(1), 171-176 (2004).

[11] Roundy, S. and Wright, P. K., "A piezoelectric vibration based generator for wireless electronics," Smart Materials and structures 13(5), 1131 (2004).

[12] Ottman, G. K., Hofmann, H. F., Bhatt, A. C., and Lesieutre, G. A., "Adaptive piezoelectric energy harvesting circuit for wireless remote power supply," Power Electronics, IEEE Transactions on 17(5), 669-676 (2002).

[13] Beeby, S. P., Tudor, M. J., and White, N., "Energy harvesting vibration sources for microsystems applications," Measurement science and technology 17(12), R175 (2006).

[14] Ferrari, M., Ferrari, V., Marioli, D., and Taroni, A., "Modeling, fabrication and performance measurements of a piezoelectric energy converter for power harvesting in autonomous microsystems," IEEE transactions on instrumentation and measurement 55(6), 2096-2101 (2006).

[15] Ferrari, M., Ferrari, V., Guizzetti, M., and Marioli, D., "An autonomous battery-less sensor module powered by piezoelectric energy harvesting with rf transmission of multiple measurement signals," Smart materials and Structures 18(8), 085023 (2009).

[16] Al-Ashtari, W., Hunstig, M., Hemsel, T., and Sextro, W., "Frequency tuning of piezoelectric energy harvesters by magnetic force," Smart Materials and Structures 21(3), 035019 (2012).

[17] Mann, B. and Sims, N., "Energy harvesting from the nonlinear oscillations of magnetic levitation," Journal of Sound and Vibration 319(1), 515-530 (2009).

[18] Stanton, S. C., McGehee, C. C., and Mann, B. P., "Nonlinear dynamics for broadband energy harvesting: Investigation of a bistable piezoelectric inertial generator," Physica D: Nonlinear Phenomena 239(10), 640$653(2010)$.

[19] Stanton, S. C. and Mann, B. P., "Harvesting energy from the nonlinear oscillations of a bistable piezoelectric inertial energy generator," in [ASME 2009 International Design Engineering Technical Conferences and Computers and Information in Engineering Conference], 447-456, American Society of Mechanical Engineers (2009).

[20] Ferrari, M., Ferrari, V., Guizzetti, M., Andò, B., Baglio, S., and Trigona, C., "Improved energy harvesting from wideband vibrations by nonlinear piezoelectric converters," Procedia Chemistry 1(1), 1203-1206 (2009).

[21] Ferrari, M., Ferrari, V., Guizzetti, M., Andò, B., Baglio, S., and Trigona, C., "Improved energy harvesting from wideband vibrations by nonlinear piezoelectric converters," Sensors and Actuators A: Physical 162(2), 425-431 (2010).

[22] Ferrari, M., Bau, M., Guizzetti, M., and Ferrari, V., "A single-magnet nonlinear piezoelectric converter for enhanced energy harvesting from random vibrations," Sensors and Actuators A: Physical 172(1), 287-292 (2011).

[23] Barton, D. A., Burrow, S. G., and Clare, L. R., "Energy harvesting from vibrations with a nonlinear oscillator," Journal of Vibration and Acoustics 132(2), 021009 (2010).

[24] Vocca, H., Neri, I., Travasso, F., and Gammaitoni, L., "Kinetic energy harvesting with bistable oscillators," Applied Energy 97, 771-776 (2012).

[25] Tang, L., WU, H., YANG, Y., and SOH, C. K., "Optimal performance of nonlinear energy harvesters," in [22nd International Conference on Adaptive Structures and Technologies], (2011).

[26] Tang, L., Yang, Y., and Soh, C.-K., "Improving functionality of vibration energy harvesters using magnets," Journal of Intelligent Material Systems and Structures, 1045389X12443016 (2012). 
[27] Tang, L. and Yang, Y., "A nonlinear piezoelectric energy harvester with magnetic oscillator," Applied Physics Letters 101(9), 094102 (2012).

[28] Younis, M. I., [MEMS linear and nonlinear statics and dynamics], vol. 20, Springer Science \& Business Media (2011). 